\title{
International Journal of Sciences
}

Research Article

Volume 7 - May 2018 (05)

\section{Evaluation of Maxillary Sinus Floor Augmentation on Digital Panoramic Radiographs with an Image Manipulation Programme after Simultaneous or Staged Dental Implant Insertion*}

\author{
Walter Sutter ${ }^{1}$, Marius Meier², Sebastian Berger ${ }^{1}$, Klaudio Pauli' ${ }^{1}$, \\ Fabian Arnosti ${ }^{3}$, Vanessa Werth ${ }^{4}$, Dritan Turhani ${ }^{1}$
}

\author{
${ }^{1}$ Centre for Oral and Maxillofacial Surgery, University of Dental Medicine and Oral Health, Danube Private \\ University, Krems, Austria \\ ${ }^{2}$ Private Surgery, Vaduz, Lichtenstein \\ ${ }^{3}$ Private Surgery, Bad Ragaz, Swiss \\ ${ }^{4}$ Private Surgery, Mauren, Lichtenstein
}

*The materials used in this study and all funding support were provided by the Danube Private University, Krems, Austria.

\begin{abstract}
Sinus floor augmentation is an effective method to regain bone height for the successful insertion of dental implants into the posterior maxilla. The aim of the study was to evaluate the behaviour of augmentation material following simultaneous or staged dental implant insertion, as visible on digital panoramic radiographs using the GNU Image Manipulation Program (GIMP). We evaluated one-stage (group 1) or two-stage (group 2) maxillary sinus floor augmentation procedures in 19 patients, using a high temperature-treated bovine porous hydroxyapatite material. Digital panoramic radiographic measurements were captured pre-operatively in both groups, pre-implant insertion in group 2, and immediately postoperatively and 6 months postoperatively in both groups. Forty parallelwalled bone-level implants were placed in a one-stage $(n=18)$ or two-stage $(n=22)$ protocol, with a mean residual bone height of $4.9 \pm 1.8 \mathrm{~mm}$ and survival rate of $100 \%$. Mean bone height increased by $8.6 \pm 1.6 \mathrm{~mm}$ immediately post-implantation and by $7.9 \pm 1.7 \mathrm{~mm}$ after 6 months. Mean distal and mesial bone losses after abutment connection were $0.42 \pm 0.24 \mathrm{~mm}$ and $0.34 \pm 0.27 \mathrm{~mm}$, respectively. No significant intergroup or intragroup differences between simultaneous and staged dental implant procedures were found. Our results show that the histogram tool in GIMP is useful for documentation of the area of the augmentation material used in maxillary sinus floor augmentation.
\end{abstract}

Keywords: Dental Implants; Radiographic Image Interpretation, Computer-Assisted; GNU Image Manipulation Program (GIMP), Sinus Floor Augmentation

\section{Introduction:}

Dental implants are widely used and have a good long-term survival rate (E. Jung et al., 2012; Pjetursson et al., 2012). Further, maxillary sinus floor augmentation is a reliable method for increasing bone height in the posterior maxilla (Del Fabbro et al., 2008; Danesh-Sani et al., 2016), and can be performed via either of two approaches; external sinus lift (lateral access) (Boyne and James, 1980) or internal sinus lift (transalveolar access) (Summers, 1994), with or without use of augmentation material (Silva et al., 2016). When an augmentation material is required, an allograft, alloplastic material, or xenograft can be used (Esposito et al., 2014) to avoid the disadvantages of autogenous bone, which include increased morbidity, limited availability, and high volumetric change (Papageorgiou et al., 2016; Wu et $a l ., 2016)$. Using lateral access, closure of the lateral window with a membrane and fixation with tensionfree seams after augmentation is recommended (Garg and Quinones, 1997; Wallace and Froum, 2003; Suarez-Lopez Del Amo et al., 2015).

This article is published under the terms of the Creative Commons Attribution License 4.0 Author(s) retain the copyright of this article. Publication rights with Alkhaer Publications. Published at: http://www.ijsciences.com/pub/issue/2018-05/

DOI: 10.18483/ijSci.1658; Online ISSN: 2305-3925; Print ISSN: 2410-4477 
Dental implants can be inserted in two stages, whereby a 4-6-month healing period is allowed between sinus floor augmentation and insertion of the implant. However, this strategy has some disadvantages, including a long wait time until final prosthetic restoration, a need for two surgical procedures, and the expense for patients (Al-Nawas and Schiegnitz, 2014; Esposito et al., 2014). A onestage surgical technique whereby the implant is inserted immediately after the surgical sinus lift procedure in one sitting has been introduced as a more effective treatment. No differences in implants placed using a one-stage or two-stage technique have been observed (Esposito et al., 2014; Felice et al., 2014). However, the risk of implant failure has been reported to be higher if there is a residual bone height below the maxillary sinus of $1-3 \mathrm{~mm}$ when a onestage procedure is performed (Felice et al., 2014).

In many studies of maxillary sinus floor augmentation techniques and implant placement, histological and histomorphometric analysis of the newly formed bone has required a bone biopsy to be taken preoperatively (Danesh-Sani et al., 2016). However, digital panoramic radiography, computed tomography, and/or cone beam computed tomography (CBCT) can now be used for noninvasive measurement of augmentation status before and after implantation (Shanbhag et al., 2014; Danesh-Sani et al., 2016). The market for CBCT has expanded markedly in recent years (Jacobs and Esposito, 2016). However, the radiation burden of CBCT is considerably higher than that of conventional two-dimensional (2D) radiography (Harris et al., 2012), and users of CBCT are heavily dependent on the manufacturer because the system is essentially a closed one (Friedland and Metson, 2014). Further, a method for assessment of change in the behaviour of the augmentation material that is easy to perform in routine clinical practice using digital panoramic radiography has yet to be established.

Accurate quantification of bone regeneration on digital radiography images is now possible using the GNU Image Manipulation Program (GIMP 2.8.18), which is freely available for analysis of digital images (Schonberger et al., 2010). GIMP contains several tools that are useful for enhancement and investigation of features seen on panoramic images (alyona; Kim TG, Lee YS, Kim YP, Park YP, Cheon MW, 2014; 22: 361-368; Carolina Sparavigna, 2015), in particular changes in alveolar bone density around dental implants (Ramachandran et al., 2016).

The aim of this study was to examine digital panoramic radiographs using the histogram tool of GIMP for changes in the porous hydroxyapatite material used in one-stage and two-stage sinus floor augmentation procedures during the initial healing period (up to 6 months; Delilbasi and Gurler, 2013).

\section{Material and Methods \\ Patients}

The patient database at the Centre for Oral and Maxillofacial Surgery, Danube Private University, Krems, Austria, was retrospectively reviewed to identify all sinus floor augmentation procedures performed from 2013 to 2015 . These procedures were then classified into two groups, according to whether sinus floor augmentation and implantation were undertaken simultaneously (one-stage technique, group 1) or in separate sessions (two-stage technique, group 2). Digital panoramic radiography (Orthophos SL 3D; Dentsply Sirona, Himmelreich, Austria) was performed in all patients prior to the surgical procedures being undertaken. All patients were then informed verbally and in writing about the risks, potential side effects, alternative treatment methods available, and the consequences of not undergoing surgery. By signing a formal anamnesis questionnaire, the patients agreed the use of their data in this study which were conducted according to the World Medical Association (WMA) Declaration of Helsinki - Ethical Principles for Medical Research. Further the study protocol was approved by the central Ethical Review Board of the federal state of Lower Austria (approval number GS4-EK4/451-2017).

The study inclusion criteria were as follows: availability of preoperative digital panoramic radiographs (groups 1 and 2); availability of digital panoramic radiographs immediately after implantation (group 1); availability of digital panoramic radiographs 6 months after sinus floor augmentation and prior to implantation (group 2); and availability of digital panoramic radiographs 6 months after implantation (groups 1 and 2).

Twenty-three sinus floor augmentation procedures performed in 19 patients (six women, 13 men; age range 31-68 years) were eligible for inclusion in the study. Thirteen surgical procedures involved the left side, 10 involved the right side, and three patients underwent bilateral sinus floor augmentation. The medical history was unremarkable in 12 patients; in the remaining seven patients, the history included medical treatment for hypothyroidism $(n=2)$ or hyperthyroidism $(\mathrm{n}=1)$, documented hypertension $(n=2)$, penicillin allergy $(n=2)$, antidepressant therapy $(\mathrm{n}=1)$, and asthma $(\mathrm{n}=1)$.

\section{Surgical protocol}

All the surgical procedures were performed by the same surgeon (DT) during the study period. Prior to each surgical procedure, oral disinfection was performed by rinsing with chlorhexidine-digluconate 
solution 2\% (GlaxoSmithKline Pharma, Vienna, Austria) for 1 minute. Surgery was performed under local anaesthesia using Ultracain D-S Forte (Sanofi, Vienna, Austria) or $2 \%$ Xylocaine Dental with epinephrine 1:50,000 (Dentsply Sirona). In both groups, an alveolar crest incision was performed, followed by elevation of a full-thickness flap to expose the posterior and lateral maxillary area and creation of a lateral bone window using diamond burs (Komet Dental, Lemgo, Germany). The Schneiderian membrane was elevated carefully using conventional sinus lift instruments (Hu-Friedy Mfg. Co., LLC. Tuttlingen, Germany) while taking care to avoid perforating the membrane. If a perforation did occur, the elevation was extended in all directions, and the defect in the sinus membrane was covered using a fibrinogen-thrombin-collagen patch (TachoSil®; Takeda, Linz, Austria). This membrane was applied underneath the Schneiderian membrane to prevent dislocation of the grafting material.

The bone substitute material (Endobon ${ }^{\circledR}$ Xenograft Granules; Zimmer-Biomet, Palm Beach Gardens, FL, USA) was rehydrated in sterile saline solution and placed into the newly created space between the fibrinogen-thrombin-collagen membrane and the sinus floor. When the one-stage technique was used, the implant positions were marked with a round bur, and drilling for site preparation followed the surgical protocol recommended by Zimmer-Biomet for a parallel-walled bone-level implant (T3 [n=2], Osseotite Certain 2 [n=2], T3 Non-Platform switched $[n=14])$. All implants demonstrated good primary stability and a healing cap was used. A second membrane was placed on the bone substitute material exposed on the lateral aspect of the sinus. The mucoperiosteal flap was positioned and sutured using saliva-proof polypropylene single sutures (Perma Sharp 4.0 or 5.0; Hu-Friedy), and then allowed to heal for 3 months. A panoramic radiograph was taken preoperatively as a radiological control. When a twostage technique was used, a standard implant (T3 [n=9] or T3 Non-Platform switched implant [ $n=13]$ ) was inserted and a panoramic radiograph was taken as a radiological control at the end of the 6-month healing period.

All patients received amoxicillin/clavulanic acid $1 \mathrm{~g}$ orally twice daily or clindamycin $300 \mathrm{mg}$ orally three times daily for 7 days as antibiotic prophylaxis, and were advised to avoid sneezing and to use a nasal spray when required. A nonsteroidal antiinflammatory agent was prescribed for analgesia during the week following surgery. All patients were reviewed and had their sutures removed after 10-12 days. No postoperative antimicrobial rinses were prescribed. Mild postoperative oedema and surgical wound pain were noted. No infections or other complications were recorded.

\section{Implants and prosthetic technique}

Bone-level parallel wall implants (T3 parallel wall implant, Osseotite Certain 2 Implant and T3 NonPlatform switched parallel wall implant; ZimmerBiomet) were inserted, using a one-stage or two-stage technique. Of 40 implants installed, 26 were $3.25 \mathrm{~mm}$ in diameter, one was $4.0 \mathrm{~mm}$ in diameter, and $13 \mathrm{had}$ a mean diameter of $4 \mathrm{~mm}$ with a mean Platform switch diameter of $3 \mathrm{~mm}$ (Table 1). Four patients underwent bilateral sinus surgery and 15 patients underwent a unilateral procedure. In all cases, long implants $(8.5,10$, and $11.5 \mathrm{~mm})$ were used according to the amount of newly formed bone at the site of the implant. Routine prosthetic procedures were undertaken after the 6-month healing period. In 15 cases, a single crown restoration was connected to the implant and in 25 cases a bridge restoration was performed. All restorations were screw-retained; 12 constructions were metal/resin crowns and 28 were zirconium/ceramic crowns. The access holes through the occlusal surfaces were closed with composite fillings (G-aenial anterior/posterior; GC Europe, Leuven, Belgium).

\section{Radiographic reference lines}

GIMP is a free open-source software application used for processing images and free-form drawing (Carolina Sparavigna, 2015; Requena-Mendez et al., 2015). Using this software, it is possible to analyse digital panoramic radiographs. For the measurements taken in this study, the original digital panoramic radiographs were exported without compression from Sidexis 4 imaging software (Dentsply Sirona) as tagged image file format files (Varma, 2012). During this process, all patient data were anonymised by the removal of patient data (DICOM; Digital Imaging and Communications in Medicine).

The radiographic reference lines are shown in colour in Figure 1. These reference lines are the same as those used in the studies by $\mathrm{Si}$ et al. (Si et al., 2013; Si et al., 2016). First, the implant axis $\{a\}$ is drawn in green. The next two lines $(\{\mathrm{e}\}$ and $\{\mathrm{b}\})$ are rotated by $90^{\circ}$ from line $\{a\}$ and pushed to the end points of the implant. The jaw is drawn in red (mesial $\{\mathrm{cm}\}$, distal $\{\mathrm{cd}\})$. The blue lines $\{\mathrm{dd}\}$ and $\{\mathrm{dm}\}$ are, respectively, mesial and distal to the bony sinus floor. The apical bone line after sinus augmentation is marked as $\{\mathrm{f}\}$.

\section{Standard radiographic evaluation}

The radiographs were selected from the database at the Centre for Oral and Maxillofacial Surgery, University of Dental Medicine and Oral Health, Danube Private University, Krems, Austria. During the study period, the same operator acquired all the radiographs taken in our dental department and all patients were positioned with the head oriented in the Frankfurt horizontal plane parallel to the floor. All 
radiographs were acquired using a $2 \mathrm{D} / 3 \mathrm{D}$ hybrid $\mathrm{X}$ ray unit (Orthophos SL 3D). The radiographic settings included a digital cadmium-telluride sensor with direct conversion sensor technology measuring $146 \mathrm{~mm} \times 6 \mathrm{~mm}$ with a resolution of $100 \mu \mathrm{m} /$ pixel, a tube voltage of $60-90 \mathrm{kV}$, and a current of 3-16 mA, with $14.4 \mathrm{~s}$ of exposure time and an effective (Ludlow) dose for high definition of 57-273 $\mu \mathrm{Sv}$.

The digital panoramic radiographs were used to obtain the following measurements: (1) the height of the residual bone at each implant site $(\mathrm{RBH}) ;(2)$ the height of the newly formed bone in the maxillary sinus after 6 months; and (3) the marginal bone-level changes around each implant. The distance from the shoulder of the implant to the first bone-to-implant contact (DIB) was measured. The time of the abutment connection was set as the baseline for measurement of the distance from the implant shoulder to the DIB. If a vertical bone defect was observed, the most apical level of the defect was measured.

As shown in Figure 2, the following values were measured:

- the residual bone height $(\mathrm{RBH})$ as an average measured by mesial $\left(\mathrm{RBH}_{\mathrm{m}}\right)$ and distal $\left(\mathrm{RBH}_{\mathrm{d}}\right)$; the distance from the intersection implant to $\{\mathrm{cm}\}$ to $\{\mathrm{dm}\}$ or intersection of the implant with $\{c d\}$ to $\{d d\}$ parallel to $\{a\}$

- the distance from the intersection $\{a \mathrm{a}\}$ to the apical $\{$ af $\}$ parallel to $\{a\}$

- the implant length, measured from $\{b\}$ to $\{e\}$ parallel to $\{a\}$

- the implant protrusion (IPL) as an average measured by mesial (IPL $)$ and distal $\left(\mathrm{IPL}_{\mathrm{d}}\right)$; the distance from the intersection implant to the $\{\mathrm{dm}\}$ to $\{\mathrm{e}\}$ and intersection implant with $\{\mathrm{dd}\}$ to $\{\mathrm{e}\}$ parallel to $\{\mathrm{a}\}$

- apical bone height as the distance between $\{\mathrm{e}\}$ and $\{\mathrm{f}\}$ measured on $\{\mathrm{a}\}$

- the crestal bone level (CBL) as an average measured by mesial $\left(\mathrm{CBL}_{\mathrm{m}}\right)$ and distal $\left(\mathrm{CBL}_{\mathrm{d}}\right)$; the distance from the intersection implant to $\{\mathrm{cm}\}$ to $\{b\}$ and intersection of the implant with $\{c d\}$ to $\{b\}$ parallel to $\{a\}$

- The bone growth to the implant. The sum of IPL of the initial position and the apical bone height of the respective measurement.

\section{Evaluation of radiographic area}

The original digital panoramic radiography image was entered into the GIMP and magnified, as shown in the upper panel of Figure 1. In this manner, using the tool "free choice", the newly gained area could be framed and displayed in yellow $\{\mathrm{f}\}$. Applying the "histogram" tool, it was possible to calculate and display the area with a certain number of pixels.
Because of slight changes in patient positioning during multiple recordings, the distortion of the image had to be subtracted first by taking the inserted implants as a reference. If multiple implants were present, only the implant situated closest to the mesial aspect was taken as a reference. By means of two green lines $\{b / e\}$ parallel to $\{a\}$, the implant could be displayed in its full length. Using the "measuring tape" tool, the distance between the intersections $\{a b\}$ and $\{a e\}$ could be measured and expressed in the form of a pixel value.

Based on this value and the original length $(\mathrm{mm})$ of the implant, it was possible to determine a factor on each digital panoramic radiograph. Starting from this factor, the newly gained area of the bone replacement material could be determined by simply converting the pixel number in $\mathrm{mm}^{2}$ (factor and area calculation; Figure 2). The digital panoramic radiographs taken immediately and 6 months after the implantation procedure were also separately inserted into the GIMP. A separate factor was calculated for digital panoramic radiographs taken at each of these two time points.

\section{Statistical analysis}

All study data were tested for normality using the Kolmogorov-Smirnov adaptation test. Data were compared between the groups using the $t$-test for inter-rater reliability. All data are shown as the mean and standard deviation. Statistical analysis was performed using SPSS version 23.0 software (IBM Corp., Armonk, NY, USA). A p-value of $<0.05$ was considered to be statistically significant.

\section{RESULTS}

\section{Clinical findings}

No implants were lost during the study period (mean $26.1 \pm 9.7$ months). A perforation of the sinus mucosa occurred during sinus floor augmentation in one patient in group $2(1 / 23,4.4 \%)$. No complications occurred during the healing period. One patient in group 2 developed emphysema after the sinus lift, which resolved after 1 week. No crowns showed loosening of their assembly screws or other complications during follow-up.

\section{Radiological findings}

The mean height of the residual bone at the implant site was $5.1 \pm 2.3 \mathrm{~mm}$ in group 1 and $4.8 \pm 1.2 \mathrm{~mm}$ in group 2. The mean height of the residual bone when groups 1 and 2 were combined was $4.9 \pm 1.8 \mathrm{~mm}$. Six months later, the mean height of the newly formed bone in the maxillary sinus was $7.7 \pm 1.3 \mathrm{~mm}$ in group 1 and $8.0 \pm 1.5 \mathrm{~mm}$ in group 2 . The mean height of the newly formed bone in the maxillary sinus when groups 1 and 2 were combined was $7.9 \pm$ $1.7 \mathrm{~mm}$. The distance from the implant shoulder to the most coronal bone-to-implant contact (DIB) was 
measured at the time of the abutment connection; the mean distal and mesial bone loss was $0.42 \pm 0.24 \mathrm{~mm}$ and $0.34 \pm 0.27 \mathrm{~mm}$, respectively.

\section{Statistical findings}

When groups 1 and 2 were combined, there was no statistically significant difference between the mean area measured immediately after implantation and that measured 6 months later $\left(82.4 \pm 33 \mathrm{~mm}^{2}\right.$ vs 79.2 $\pm 30.7 \mathrm{~mm}^{2} ; \mathrm{p}=0.092$, $t$-test). There was also no statistically significant difference between groups 1 and 2 with regard to the mean areas measured immediately after implantation $\left(88.9 \pm 33.8 \mathrm{~mm}^{2}\right.$ vs $76.5 \pm 32.5 \mathrm{~mm}^{2} ; \mathrm{p}=0.379, t$-test $)$ or at 6 months after implantation $\left(85.2 \pm 30.7 \mathrm{~mm}^{2}\right.$ vs $73.7 \pm 30.9 \mathrm{~mm}^{2}$; $\mathrm{p}=0.372, \quad t$-test). Figure 3 shows the area measurements as a box-and-whisker plot.

\section{Discussion}

Sinus floor augmentation is a necessary standard preprosthetic surgical procedure for insertion of dental implants in patients with advanced atrophy of the maxillary alveolar ridge (Danesh-Sani et al., 2016), and use of grafting materials for this purpose is predictable and reliable (Silva et al., 2016).

The lateral window technique requires a minimum residual bone height of $4-5 \mathrm{~mm}$ as a threshold for simultaneous sinus floor elevation to obtain sufficient primary stability of the implant without disturbing the osseointegration process (Del Fabbro et al., 2008), which is easier to obtain after two treatment sessions. However, the reliability of a one-step method and the behaviour of the augmentation material after implantation are of considerable interest. A healing period of approximately 6 months has been the standard of care for implants placed with simultaneous sinus floor elevation in the past 25 years (Checchi et al., 2010; Kahnberg et al., 2011; Rasmusson et al., 2012), and meets the demands for a short treatment time and reliable results. However, there is always the question of how to document and measure the results of treatment so that any postoperative changes in the area of the augmentation material can be identified.

In the present study, we sought to find a way of obtaining reliable measurements of bone augmentation that would be both easy to perform and simply integrated into routine clinical practice.

Standard non-invasive methods for radiological measurement of augmentation status before and after dental implantation include digital panoramic radiography, computed tomography, and CBCT (Shanbhag et al., 2014; Danesh-Sani et al., 2016). With the ongoing technological improvements in the quality of radiography, more detailed 2D panoramic images have become available. Because of the lower costs, 2D radiography still seems to be the standard for many practitioners for documentation and followup purposes.

In 2015, Sparavigna recommended use of the GIMP for enhancement of panoramic radiographs (Carolina Sparavigna, 2015). There are numerous fields of application for computer graphic software programmes like GIMP or Adobe Photoshop (Adobe Systems Inc., San Jose, CA, USA). These programmes have several tools that can be useful for investigation of details of panoramic images (Kim TG, Lee YS, Kim YP, Park YP, Cheon MW, 2014; 22: 361-368). Processing of the images can increase the visibility of details by simply adjusting the brightness and contrast or using other methods available in the programme tools (Solomon, 2009; Carolina Sparavigna, 2015). One of the most commonly used tools is the histogram function which analyses differences in grayscale values (Hoppe et al., 2013; Carolina Sparavigna, 2015; Meshram et al., 2015).

Changes in alveolar bone density around a dental implant seem to be particularly amenable to investigation using these programmes (Ramachandran et al., 2016). Use of GIMP has also been reported in several other dental indications. Alzahrani et al. examined the influence of plateletrich fibrin on post-extraction socket healing (Alzahrani et al., 2017). Further, the success of osseous regeneration after enucleation of cystic lesions can be examined (Meshram et al., 2015). In forensic odontology, the need to estimate the age of living adults has increased, and panoramic and periapical radiographs have been used to evaluate the correlation between the tooth and pulp to facilitate estimation of dental age (Azevedo et al., 2015). In a cariology study, bitewing radiographs were analysed to quantitatively determine the grayscale value of the affected dentin beneath restorations and compare it with that of healthy dentin (Kielbassa et al., 2017). External and internal infiltration of resin into natural proximal subsurface carious lesions was analysed using confocal laser microscopy and GIMP as a dedicated image manipulation programme (Kielbassa et al., 2017). These programmes can also be used for shade-taking, shade analysis, and creation of the final restoration (McLaren et al., 2017), or to ascertain tooth colour, e.g., when assessing the effectiveness of bleaching (Zanjani et al., 2015).

A number of studies in medicine have also used GIMP for measurement purposes and to improve the ability to recognise pathological changes, in particular for robust and serial evaluation of breast radiographs. GIMP can also be used to evaluate changes in the echogenicity of tumour tissue after 
chemotherapy (Lin et al., 2013).

The quality of panoramic radiographs improved when the Retinex filter was utilised in the GIMP software (Carolina Sparavigna, 2015) In 2009, Solomon compared GIMP with Adobe Photoshop, and concluded that both programmes are similar in their processing abilities and that a good processing programme need not be expensive (Solomon, 2009). Therefore, we elected to use GIMP in our research.

In this study, area measurements were analysed using the histogram tool in the image analysis software. The mean pixel grayscale values obtained with the aid of the histogram range from 0 (completely black appearance resulting from the total absence of pixels) to 255 (completely white appearance resulting from the presence of the maximum possible pixel density). Referenced on the original implant length $(\mathrm{mm})$, it was possible to determine a specific factor for each panoramic radiograph. Starting from this factor, the newly gained area of the bone replacement material could be determined by simply converting the pixel number to $\mathrm{mm}^{2}$. Using this method, it was easy to measure the initial bone height and the augmented area in regard to the implants and the changes in the augmentation material in respect to bone formed in the maxillary sinus after 6 months. Distal and mesial crestal bone loss could also be observed. Alzahrani et al. used a similar approach in their study of the effect of platelet-rich fibrin on post-extraction socket healing (Alzahrani et al., 2017), in which they measured the surface area of the extraction sockets using Adobe Photoshop according to the method described by Chiapasco et al. (Chiapasco et al., 2000). First, the radiographic images were transferred to grayscale tonalities of 256 . The extraction socket was then marked and converted to a histogram and finally calculated in millimetres (Alzahrani et al., 2017). A further study by Meshram et al. used a grayscale histogram for radiological follow-up of osseous regeneration after cystic enucleation (Meshram et al., 2015). GIMP and other programmes like Adobe Photoshop have a wide range of applications. The histogram tool provides an opportunity for follow-up of bone healing or, in our case, the behaviour of bone replacement material (Hieu et al., 2010).

A number of studies have examined whether there are any postoperative changes in maxillary sinus volume or the dimensions of the grafts used in maxillary sinus augmentation (Kirmeier et al., 2008; Zijderveld et al., 2009; Hieu et al., 2010; Cosso et al., 2014; Favato et al., 2015; Alzahrani et al., 2017). Hieu et al. evaluated the changes in height of grafts containing xenogenic materials on radiographs and reported significant resorption of these materials over a 2-year period (Hieu et al., 2010; Shanbhag et al.,
2014). The air pressure in the maxillary sinus, the augmentation material and membranes used, and factors such as bone density, implant material, surface quality, and implant length and width may influence the results (Degidi et al., 2013; Felice et al., 2014; Shanbhag et al., 2014). Wanschitz et al. reported an approximate mean loss in volume of the bone replacement material of $13.9 \% \pm 1.9 \%$; (Wanschitz et al., 2006) depending on the material used, the contraction in volume has ranged from $25 \%$ to $40 \%$ (Hieu et al., 2010; Cosso et al., 2014). Other studies of force loading on dental implants concluded that implant loading has a positive effect in terms of the ability of the graft to maintain its height (Nyström et al., 1993; Hieu et al., 2010). The reasons for the mean distal and mesial bone loss could be related to the remodelling phase postoperatively (Kahnberg et al., 2011). In our present study, there was no significant difference in the measured areas between the one-stage and two-stage techniques immediately after implantation or 6 months later. This finding is in line with that of previous studies that have compared the one-stage and two-stage techniques (Kahnberg et al., 2011; Degidi et al., 2013), and confirms the usefulness of the histogram tool in GIMP for documentation of the behaviour of the augmentation material used during sinus lift procedures.

\section{Conclusion}

Image manipulation programmes have a range of potential applications. Within the limitations of digital panoramic radiographs, these programmes afford an opportunity to obtain area measurements of bone augmentation material. In this study, we found no significant difference in the measured area between the one-stage and two-stage techniques immediately after implantation or at follow-up period of 6 months. GIMP allows reliable augmentation graft measurements to be obtained easily. Further, the software is freely available, increasing its potential for implementation in routine dental practice.

\section{Acknowledgements}

The materials used in this study and all funding support were provided by the Danube Private University, Krems, Austria.

Declarations of interest: none. 


\section{Figure Captions}

Figure 1 (a) Newly gained area framed and displayed in green. (b) The "histogram" tool showing the number of pixels $(10,251)$ within the area bordered in green on the left side. Using the "measuring tape" tool, the distance between the two red dots could be measured and expressed in the form of a pixel value (117.1 pixels).
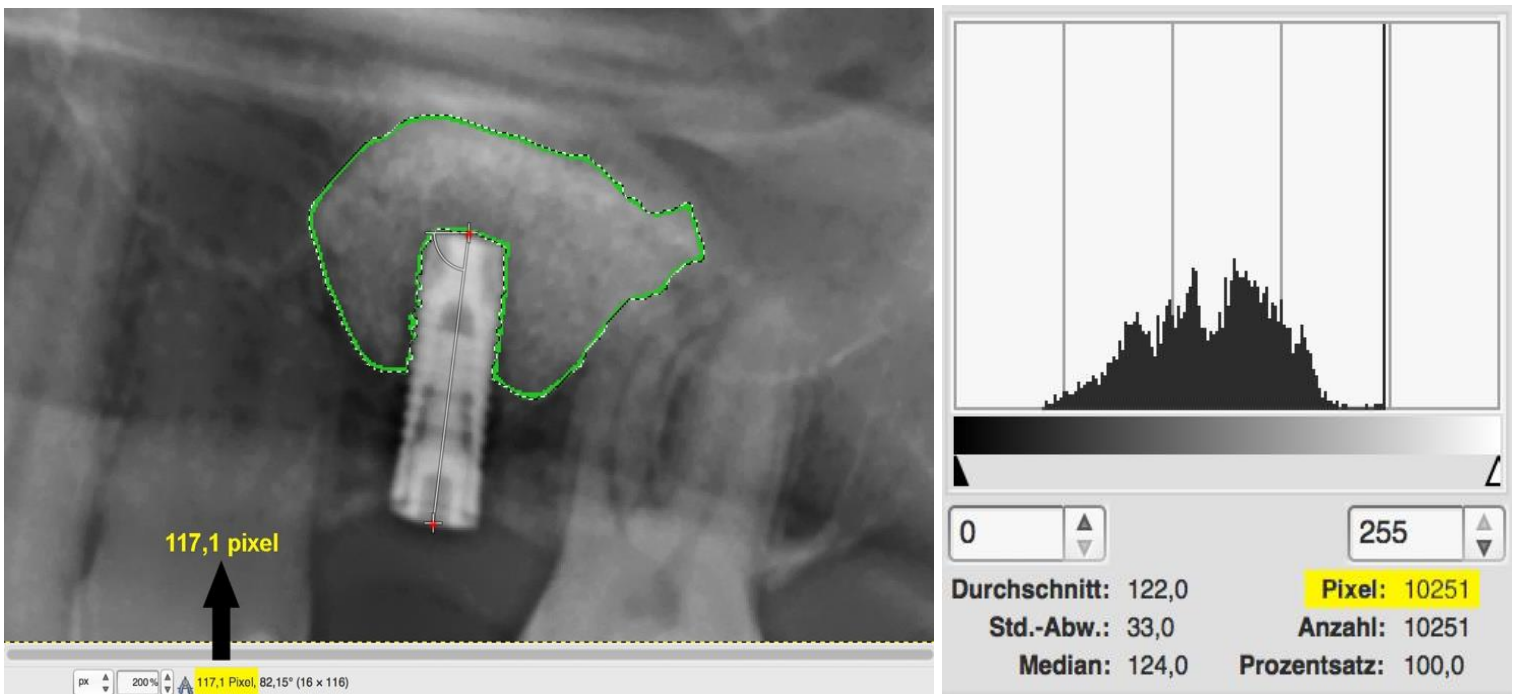

Newly gained area framed and displayed in green colour. The tool "histogram" on the right side shows the certain number of pixels (10251) within the green boardered area. Using the tool "measuring tape" the distance between the two red dots could be measured and expressed in form of a pixel value $(117,1)$.

Fig. 1 Measurements and marks in GIMP

\section{Example for factor calculation:}

Implant length real $=10 \mathrm{~mm}$

Implant length in pixel (GIMP) $\quad=117,1 \mathrm{px}$

How long is a pixel (px) in GIMP $=\mathbf{1} \mathbf{p x}=\frac{10 \mathrm{~mm}}{117,1}=\mathbf{0}, \mathbf{0 8 5 3 9 1 7} \mathbf{~ m m}$

Example for area $\left(\mathrm{mm}^{2}\right)$ calculation:

Factor $=\mathbf{0 , 0 8 5 3 9 1 7} \mathbf{~ m m}$

Number of pixels form the measured area $=10^{\prime} 251$

How big is an area $\left(\mathrm{mm}^{2}\right)$ in GIMP $=(\mathbf{0 , 0 8 5 3 9 1 7} \mathbf{~ m m})^{2} * 10^{\prime} 251=74,75 \mathrm{~mm}^{2}$

Fig. 2 Factor (px) and area $\left(\mathrm{mm}^{2}\right)$ calculation 
Evaluation of Maxillary Sinus Floor Augmentation on Digital Panoramic Radiographs with an Image Manipulation Programme after Simultaneous or Staged Dental Implant Insertion

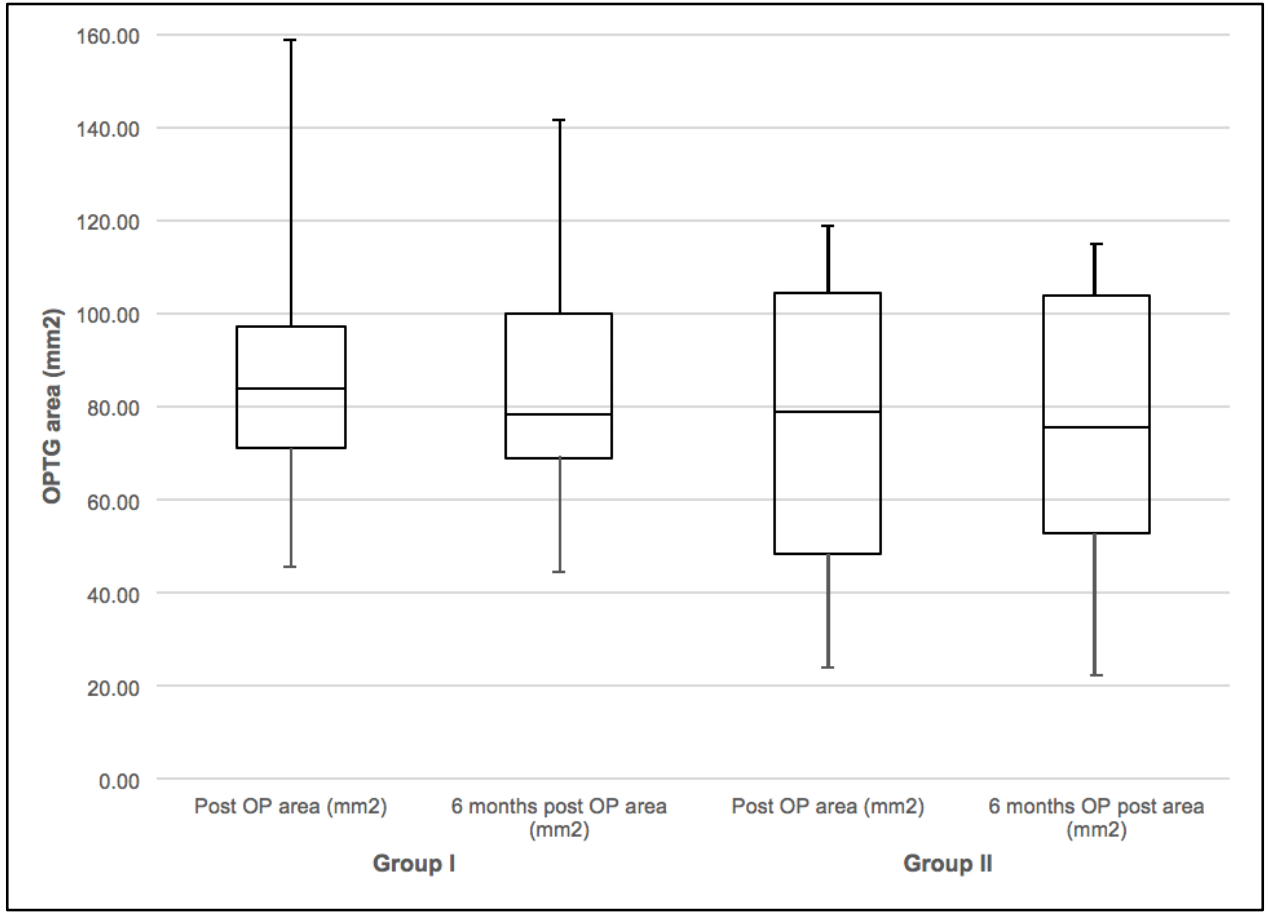

Fig. 3 Box-Whisker-Plot of the measured area $\left(\mathrm{mm}^{2}\right)$ in Group I and Group II

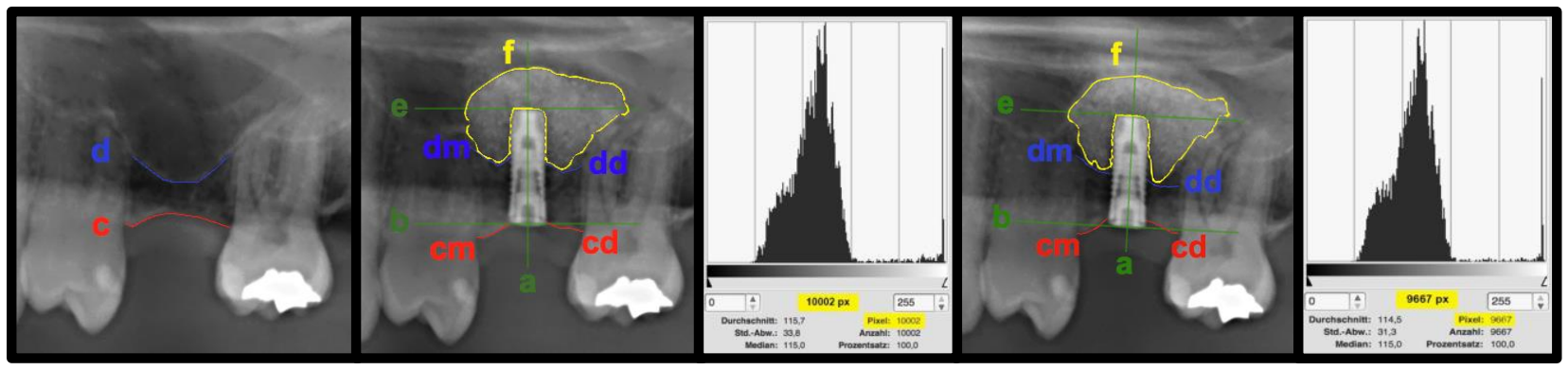

Fig. Group I;

A: Initial situation. B: Situation direct after implantation. C: Histogram direct after implantation. D: Situation 6 months after implantation. E: Histogram 6 months after implantation. 
Evaluation of Maxillary Sinus Floor Augmentation on Digital Panoramic Radiographs with an Image Manipulation Programme after Simultaneous or Staged Dental Implant Insertion

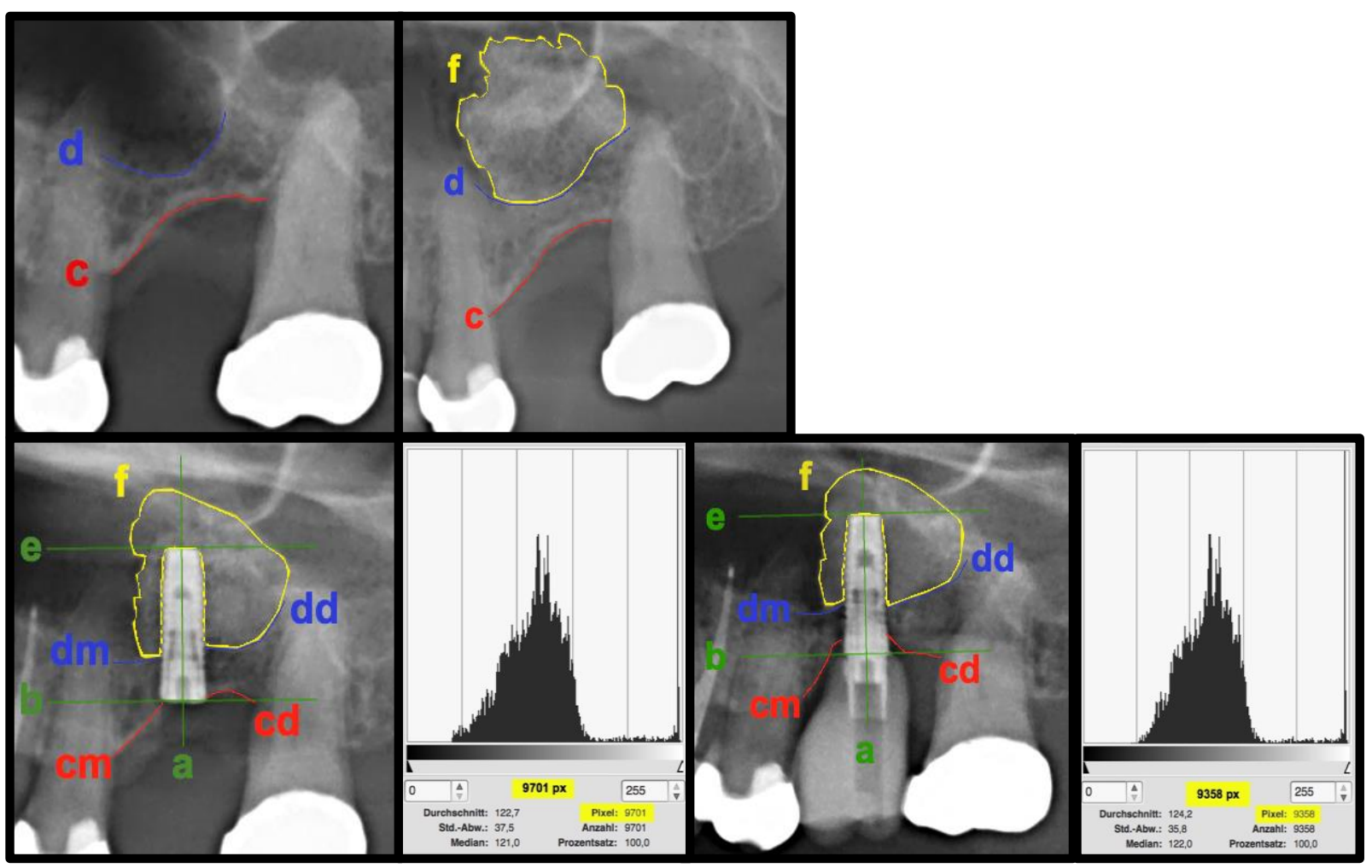

Fig. Group II;

A: Initial situation. B: Situation direct after sinus floor augmentation. C: Situation direct after implantation. D: Histogram direct after implantation.

E: Situation 6 months after implantation. F: Histogram 6 months after implantation.

Table 1 Types and locations of implants used in this study

\begin{tabular}{|c|c|c|c|c|c|c|c|c|c|c|c|}
\hline $\begin{array}{l}\text { Patient } \\
\text { number }\end{array}$ & $\begin{array}{c}\text { Group } \\
1 / 2\end{array}$ & $\begin{array}{l}\text { Number of } \\
\text { implants }\end{array}$ & $\begin{array}{c}\text { Quadrant } \\
1 / 2\end{array}$ & $\begin{array}{l}\text { Implant } \\
\text { position }\end{array}$ & $\begin{array}{l}\text { Residual bone } \\
\text { height }(\mathrm{mm})\end{array}$ & $\begin{array}{l}\text { Implant } 1 \\
(\mathrm{~mm})\end{array}$ & Implant type & $\begin{array}{c}\text { Implant } 2 \\
(\mathrm{~mm})\end{array}$ & Implant type & $\begin{array}{c}\text { Implant } 3 \\
(\mathrm{~mm})\end{array}$ & Implant type \\
\hline 1 & 1 & 1 & 2 & 25 & 2.9 & $10 \times 3.25$ & OC 2 & & & & \\
\hline 2 & 1 & 1 & 1 & 15 & 5.1 & $10 \times 3.25$ & T3 NPSPW & & & & \\
\hline 3 & 1 & 2 & 1 & $15 / 16$ & 6.5 & $10 \times 3.25$ & T3 NPSPW & $10 \times 3.25$ & T3 NPSPW & & \\
\hline 4 & 1 & 1 & 1 & 15 & 4.4 & $10 \times 4$ & OC 2 & & & & \\
\hline 5 & 1 & 2 & 2 & $26 / 27$ & 8.2 & $10 \times 3.25$ & T3 NPSPW & $10 \times 3.25$ & T3 NPSPW & & \\
\hline 6 & 1 & 2 & 2 & $26 / 27$ & 4.0 & $10 \times 3.25$ & T3 NPSPW & $10 \times 3.25$ & T3 NPSPW & & \\
\hline 7 & 1 & 3 & 2 & $24 / 25 / 26$ & 9.9 & $10 \times 3.25$ & T3 NPSPW & $10 \times 3.25$ & T3 NPSPW & $10 \times 3.25$ & T3 NPSPW \\
\hline 8 & 1 & 2 & 2 & $25 / 26$ & 4.9 & $11.5 \times 3.25$ & T3 NPSPW & $10 \times 3.25$ & T3 NPSPW & & \\
\hline 9 & 1 & 2 & 2 & $25 / 26$ & 3.3 & $10 \times 4 / 3$ & T3 NPSPW & $8.5 \times 4 / 3$ & T3 NPSPW & & \\
\hline 10 & 1 & 1 & 2 & 25 & 4.4 & $10 \times 4 / 3$ & T3 PW & & & & \\
\hline 11 & 2 & 1 & 1 & 16 & 3.4 & $11.5 \times 4 / 3$ & T3 PW & & & & \\
\hline 12 & 2 & 2 & 2 & $25 / 26$ & 4.4 & $10 \times 4 / 3$ & T3 PW & $8.5 \times 4 / 3$ & T3 PW & & \\
\hline 13 & 2 & 2 & 2 & $24 / 26$ & 4.2 & $10 \times 3.25$ & T3 NPSPW & $10 \times 3.25$ & T3 NPSPW & & \\
\hline 14 & 2 & 2 & 1 & $15 / 16$ & 3.8 & $10 \times 3.25$ & T3 NPSPW & $10 \times 3.25$ & T3 NPSPW & & \\
\hline 15 & 2 & 2 & 1 & $15 / 16$ & 4.9 & $10 \times 3.25$ & T3 NPSPW & $10 \times 3.25$ & T3 NPSPW & & \\
\hline 16 & 2 & 2 & 2 & $25 / 26$ & 5.7 & $10 \times 3.25$ & T3 NPSPW & $10 \times 3.25$ & T3 NPSPW & & \\
\hline 17 & 2 & 2 & 1 & $15 / 16$ & 5.0 & $10 \times 3.25$ & T3 NPSPW & $10 \times 3.25$ & T3 NPSPW & & \\
\hline 18 & 2 & 1 & 2 & 26 & 2.8 & $10 \times 3.25$ & T3 NPSPW & & & & \\
\hline 19 & 2 & 2 & 2 & $25 / 26$ & 6.3 & $10 \times 3.25$ & T3 NPSPW & $10 \times 3.25$ & T3 NPSPW & & \\
\hline 20 & 2 & 2 & 2 & $25 / 26$ & 6.9 & $10 \times 4 / 3$ & T3 PW & $8.5 \times 4 / 3$ & T3 PW & & \\
\hline 21 & 2 & 2 & 1 & $14 / 15$ & 5.1 & $11.5 \times 4 / 3$ & T3 PW & $10 \times 4 / 3$ & T3 PW & & \\
\hline 22 & 2 & 2 & 2 & $25 / 26$ & 5.1 & $10 \times 4 / 3$ & T3 PW & $10 \times 4 / 3$ & T3 PW & & \\
\hline
\end{tabular}




\section{References}

1. Al-Nawas B, Schiegnitz E: Augmentation procedures using bone substitute materials or autogenous bone - a systematic review and meta-analysis. Eur J Oral Implantol 7 Suppl 2: S219-34, 2014. 2. Alyona c: Application of analysis approach in Noise Estimation in Panoramic $\mathrm{X}$ - rays images using image processing program (MatLab).

3. Alzahrani AA, Murriky A, Shafik S: Influence of platelet rich fibrin on post-extraction socket healing: A clinical and radiographic study. The Saudi Dental Journal, 2017.

4. Azevedo AdCS, Alves NZ, Michel-Crosato E, Rocha M, Cameriere $\mathrm{R}$ et al.: Dental age estimation in a Brazilian adult population using Cameriere's method. Braz Oral Res 29, 2015.

5. Boyne PJ, James RA: Grafting of the maxillary sinus floor with autogenous marrow and bone. J Oral Surg 38: 613 616, 1980. 6. Carolina Sparavigna A: An Image Processing Approach Based on Gnu Image Manipulation Program Gimp to the Panoramic Radiography. ijSciences 1: 57 67, 2015.

7. Checchi L, Felice P, Antonini ES, Cosci F, Pellegrino G et al.: Crestal sinus lift for implant rehabilitation: A randomised clinical trial comparing the Cosci and the Summers techniques. A preliminary report on complications and patient preference. Eur $\mathbf{J}$ Oral Implantol 3: 221 232, 2010.

8. Chiapasco M, Rossi A, Motta JJ, Crescentini M: Spontaneous bone regeneration after enucleation of large mandibular cysts: A radiographic computed analysis of 27 consecutive cases. J Oral Maxillofac Surg 58: 942-8; discussion 949, 2000.

9. Cosso MG, Brito RB de, Piattelli A, Shibli JA, Zenóbio EG: Volumetric dimensional changes of autogenous bone and the mixture of hydroxyapatite and autogenous bone graft in humans maxillary sinus augmentation. A multislice tomographic study. Clin Oral Implants Res 25: 1251 1256, 2014.

10. Danesh-Sani SA, Loomer PM, Wallace SS: A comprehensive clinical review of maxillary sinus floor elevation: Anatomy, techniques, biomaterials and complications. Br J Oral Maxillofac Surg 54: 724 730, 2016.

11. Degidi M, Daprile G, Piattelli A: Primary stability determination of implants inserted in sinus augmented sites: 1-step versus 2-step procedure. Implant Dent 22: 530 533, 2013.

12. Del Fabbro M, Rosano G, Taschieri S: Implant survival rates after maxillary sinus augmentation. Eur J Oral Sci 116: 497 506, 2008.

13. Delilbasi C, Gurler G: Comparison of piezosurgery and conventional rotative instruments in direct sinus lifting. Implant Dent 22: $662665,2013$.

14. E. Jung R, Zembic A, Pjetursson BE, Zwahlen M, S. Thoma $D$ : Systematic review of the survival rate and the incidence of biological, technical, and aesthetic complications of single crowns on implants reported in longitudinal studies with a mean follow-up of 5 years. Clin. Oral Implants Res. 23: 2 21, 2012.

15. Esposito $M$, Felice $P$, Worthington $H V$ : Interventions for replacing missing teeth: Augmentation procedures of the maxillary sinus. Cochrane Database Syst Rev: CD008397, 2014

16. Favato MN, Vidigal BCL, Cosso MG, Manzi FR, Shibli JA et al.: Impact of human maxillary sinus volume on grafts dimensional changes used in maxillary sinus augmentation: a multislice tomographic study. Clin Oral Implants Res 26: 1450 1455, 2015.

17. Felice P, Pistilli R, Piattelli M, Soardi E, Barausse C et al.: 1 stage versus 2 -stage lateral sinus lift procedures: 1-year postloading results of a multicentre randomised controlled trial. Eur J Oral Implantol 7: 65 75, 2014.

18. Friedland B, Metson R: A guide to recognizing maxillary sinus pathology and for deciding on further preoperative assessment prior to maxillary sinus augmentation. Int $\mathrm{J}$ Periodontics Restorative Dent 34: 807 815, 2014.

19. Garg AK, Quinones CR: Augmentation of the maxillary sinus: A surgical technique. Pract Periodontics Aesthet Dent 9: 211-9; quiz 220, 1997.

20. Harris D, Horner K, Grondahl K, Jacobs R, Helmrot E et al.: E.A.O. guidelines for the use of diagnostic imaging in implant dentistry 2011. A consensus workshop organized by the European Association for Osseointegration at the Medical University of
Warsaw. Clin Oral Implants Res 23: 1243 1253, 2012.

21. Hieu P-D, Chung J-H, Yim S-B, Hong K-S: A radiographical study on the changes in height of grafting materials after sinus lift: a comparison between two types of xenogenic materials. J Periodontal Implant Sci 40: 25 32, 2010.

22. Hoppe CB, Baldissera RdS, Scarparo RK, Kopper PMP, Fontanella VRC et al:: A new assessment methodology to evaluate the radiopacity of endodontic filling materials. (Keine Angabe), 2013.

23. Jacobs R, Esposito M: Editorial: On peri-implant bone level measures: To see or not to see, that is the question. Eur J Oral Implantol 9 Suppl 1: 119 121, 2016.

24. Kahnberg K-E, Wallström M, Rasmusson L: Local sinus lift for single-tooth implant. I: clinical and radiographic follow-up. Clin Implant Dent Relat Res 13: 231 237, 2011.

25. Kielbassa AM, Ulrich I, Werth VD, Schüller C, Frank W et al:: External and internal resin infiltration of natural proximal subsurface caries lesions: A valuable enhancement of the internal tunnel restoration. Quintessence Int 48: 357 368, 2017.

26. Kim TG, Lee YS, Kim YP, Park YP, Cheon MW Study on image processing of panoramic $X$-ray using deviation improvement software, 2014; 22: 361-368.

27. Kirmeier R, Payer M, Wehrschuetz M, Jakse N, Platzer S et al:: Evaluation of three-dimensional changes after sinus floor augmentation with different grafting materials. Clin Oral Implants Res 19: 366 372, 2008.

28. Lin C-y, Cao L-h, Wang J-w, Zheng W, Chen $\mathrm{Y}$ et al: Ultrasonic spectrum analysis for in vivo characterization of tumor microstructural changes in the evaluation of tumor response to chemotherapy using diagnostic ultrasound. BMC Cancer 13: 302, 2013.

29. McLaren EA, Figueira J, Goldstein RE: A Technique Using Calibrated Photography and Photoshop for Accurate Shade Analysis and Communication. Compend Contin Educ Dent 38: 106 113, 2017.

30. Meshram VS, Lambade PN, Meshram PV, Kadu A, Tiwari MS: The autologous platelet rich fibrin: A novel approach in osseous regeneration after cystic enucleation: A pilot study. Indian J Dent Res 26: 560 564, 2015.

31. Nyström E, Kahnberg KE, Albrektsson T: Treatment of the severely resorbed maxillae with bone graft and titanium implants: histologic review of autopsy specimens. Int J Oral Maxillofac Implants 8: 167 172, 1993.

32. Papageorgiou SN, Papageorgiou PN, Deschner J, Gotz W: Comparative effectiveness of natural and synthetic bone grafts in oral and maxillofacial surgery prior to insertion of dental implants: Systematic review and network meta-analysis of parallel and cluster randomized controlled trials. J Dent 48: 1 8, 2016.

33. Pjetursson BE, Thoma D, Jung R, Zwahlen M, Zembic A: A systematic review of the survival and complication rates of implant-supported fixed dental prostheses (FDPs) after a mean observation period of at least 5 years. Clin Oral Implants Res 23 Suppl 6: 22 38, 2012.

34. Ramachandran A, Singh K, Rao J, Mishra N, Jurel SK et al.: Changes in alveolar bone density around immediate functionally and nonfunctionally loaded implants. J Prosthet Dent 115: 712 717, 2016.

35. Rasmusson L, Thor A, Sennerby L: Stability evaluation of implants integrated in grafted and nongrafted maxillary bone: a clinical study from implant placement to abutment connection. Clin Implant Dent Relat Res 14: 61 66, 2012.

36. Requena-Mendez A, Aldasoro E, Munoz J, Moore DAJ: Robust and Reproducible Quantification of the Extent of Chest Radiographic Abnormalities (And It's Free!). PLoS One 10: e0128044, 2015.

37. Schonberger T, Kasten P, Fechner K, Sudkamp NP, Pearce S et al: Novel software-based and validated evaluation method for objective quantification of bone regeneration in experimental bone defects. Z Orthop Unfall 148: 19 25, 2010.

38. Shanbhag S, Shanbhag V, Stavropoulos A: Volume changes of maxillary sinus augmentations over time: A systematic review. Int J Oral Maxillofac Implants 29: 881 892, 2014. 

Programme after Simultaneous or Staged Dental Implant Insertion

39. Si M-S, Shou Y-W, Shi Y-T, Yang G-L, Wang H-M et al.: Long-term outcomes of osteotome sinus floor elevation without bone grafts: A clinical retrospective study of 4-9 years. Clin Oral Implants Res 27: 1392 1400, 2016.

40. Si M-S, Zhuang L-f, Gu Y-x, Mo J-j, Qiao S-c et al: Osteotome sinus floor elevation with or without grafting: A 3-year randomized controlled clinical trial. J Clin Periodontol 40: 396 403, 2013.

41. Silva Ld, Lima VN de, Faverani LP, Mendonca MR de, Okamoto R et al: : Maxillary sinus lift surgery-with or without graft material? A systematic review. Int J Oral Maxillofac Surg 45: 1570 $1576,2016$.

42. Solomon RW: Free and open source software for the manipulation of digital images. AJR Am J Roentgenol 192: W3304, 2009.

43. Suarez-Lopez Del Amo F, Ortega-Oller I, Catena A, Monje A, Khoshkam $\mathrm{V}$ et al:: Effect of barrier membranes on the outcomes of maxillary sinus floor augmentation: A meta-analysis of histomorphometric outcomes. Int J Oral Maxillofac Implants 30: $607618,2015$.

44. Summers RB: A new concept in maxillary implant surgery: The osteotome technique. Compendium 15: 152, 154-6, 158 passim; quiz 162, 1994.
45. Varma DR: Managing DICOM images: Tips and tricks for the radiologist. Indian J Radiol Imaging 22: 4 13, 2012.

46. Wallace SS, Froum SJ: Effect of maxillary sinus augmentation on the survival of endosseous dental implants. A systematic review. Ann Periodontol 8: 328 343, 2003

47. Wanschitz F, Figl M, Wagner A, Rolf E: Measurement of volume changes after sinus floor augmentation with a phycogenic hydroxyapatite. Int J Oral Maxillofac Implants 21: 433 438, 2006.

48. Wu J, Li B, Lin $\mathrm{X}$ : Histological outcomes of sinus augmentation for dental implants with calcium phosphate or deproteinized bovine bone: A systematic review and meta-analysis. Int J Oral Maxillofac Surg 45: 1471 1477, 2016.

49. Zanjani VA, Ghasemi A, Torabzadeh H, Jamali M, Razmavar $\mathrm{S}$ et al.: Bleaching effect of ozone on pigmented teeth. Dent Res J (Isfahan) 12: 20 24, 2015.

50. Zijderveld SA, Schulten EAJM, Aartman IHA, Bruggenkate $\mathrm{CM}$ ten: Long-term changes in graft height after maxillary sinus floor elevation with different grafting materials: radiographic evaluation with a minimum follow-up of 4.5 years. Clin Oral Implants Res 20: 691 700, 2009. 